\title{
Использование программных пакетов LAMMPS и OVITO для исследования микрокинетики СB-синтеза алюминидов никеля*
}

\author{
В.И. Иордан, И.А. Шмаков, Ю.А. Панченко
}

Алтайский государственный университет (Барнаул, Россия)

\section{Using of LAMMPS and OVITO Software Packages to Study the Microkinetics of SH-synthesis of Nickel Aluminides}

\author{
V.I. Jordan, I.A. Shmakov, Yu.A. Panchenko
}

Altai State University (Barnaul, Russia)

\begin{abstract}
В статье рассматриваются некоторые аспекты применения программного пакета LAMMPS, peaлизующего в своей основе метод молекулярной динамики (МД), с помощью которого моделируется процесс распространения волны горения по образцу в виде пакета чередующихся слоев наноразмерных кристаллических решеток из атомов $\mathrm{Ni}$ и $\mathrm{Al}$ (атомная система порядка миллиона и более атомов) с образованием алюминидов никеля в процессе CB-синтеза. Применялась конфигурация пакета LAMMPS для выполнения на многопроцессорных вычислительных системах с использованием интерфейса MPI. Кроме того, в качестве потенциала межатомного взаимодействия были использованы две разновидности модели «погруженного атома» (англ., EAM), для которых проведены вычислительные эксперименты (ВЭ). Второй программный пакет OVITO использовался для распознавания и визуализации различных типов кристаллических, квазикристаллических и других типов структур. А именно: пакет OVITO позволил распознать и рассчитать в моделируемой атомной системе процентное содержание структур ГЦК, ОЦК, ГПУ и др. Для двух разновидностей потенциала ЕАМ в качестве результатов ВЭ приведены: семейство температурных профилей вдоль слоев структуры в последовательные моменты времени (до 12 нс) и соответствующий им набор шлифов (вертикальных сечений вдоль слоев системы атомов).

Ключевые слова: пакет LAMMPS, пакет OVITO, CB-синтез, алюминид никеля, температурный профиль, волна горения, кристаллическая решетка.
\end{abstract}

The paper discusses some aspects of the application of the LAMMPS software package, which implements the molecular dynamics (MD) method and by means of MD method simulates the process of propagation of a combustion wave along a sample in the form of a stack of alternating layers of nanoscale crystal lattices of $\mathrm{Ni}$ and $\mathrm{Al}$ atoms (the atomic system is on the order of a million and more atoms) with the formation of nickel aluminides in the process of $\mathrm{SH}$ synthesis. In addition, as the interatomic interaction potential were used two versions of the "embedded atom" model (EAM) for which computational experiments (CEs) were carried out. The second software package OVITO was used to recognize and visualize various types of crystalline, quasi-crystalline and other types of structures. Namely, the OVITO package made it possible to recognize and calculate in the simulated atomic system the percentage of next types of structures: fcc, bcc, hcp and etc. For two varieties of the EAM potential, the results of the CEs include: a family of temperature profiles along the layers of the structure at successive instants of time (up to $12 \mathrm{~ns}$ ) and a corresponding set of microsections (vertical crosssections along the layers of the atomic system).

Key words: LAMMPS software package, OVITO software package, $\mathrm{SH}$ synthesis, nickel aluminide, temperature profile, combustion wave, crystal lattice.

DOI 10.14258/izvasu(2018)4-03

\footnotetext{
*Работа выполнена при финансовой поддержке РФФИ (гранты № 18-41-220004 и № 18-08-01475).
} 


\section{Введение}

При получении современных функциональных материалов с заданными свойствами методом «самораспространяющегося высокотемпературного синтеза (CBC)» используются реагенты в виде мелкодисперсных порошков, тонких пленок, жидкостей, газов и др. Под воздействием теплового импульса в прессованном образце происходит экзотермическая реакция горения в тонком слое взаимодействующих реагентов, которая самопроизвольно распространяется по образцу путем теплопередачи от слоя к слою. На устойчивость движения фронта волны горения оказывает влияние в некоторой степени случайное распределение исходных реагентов в структуре порошковой смеси $[1,2]$. По этой причине квазипериодически меняются температура, скорость и направление распространения волны горения. «Проблема дискретности» СВС (наличие в структуре волны «микроочагов» горения) в большинстве случаев решается экспериментальным путем потому, что выводы различных теоретических моделей о поведении волны горения на макроскопическом и/или микроскопическом уровне противоречат друг другу. Для анализа микрогетерогенной структуры волны горения (эволюции дискретного распада тепловой структуры волны СВС) в локально неустойчивых режимах микрогетерогенного горения необходимо детальное изучение и уточнение физико-химических и математических модельных представлений о процессе СВ-синтеза $[3,4]$.

В статье рассмотрены результаты моделирования режимов микрогетерогенного горения при протекании процесса СВС в модельных «слоистых» структуpax порошковой смеси $\mathrm{Ni}-\mathrm{Al}$ (рис. 1) с помощью компьютерного моделирования процесса СВС методом «молекулярной динамики (МД)» в пакете LAMMPS с использованием параллельных вычислений [5].

Методические особенности моделирования СВ-синтеза алюминида никеля методом МД в пакете LAMMPS

В методе МД используются ньютоновская механика и дискретно-континуальный подход моделирова- ния эволюции системы. А именно: процесс эволюции системы частиц (как материальных точек) моделируется интегрированием уравнений движения отдельных частиц на основе простого потенциального взаимодействия с постоянным временным шагом. При моделировании на атомном уровне для уточнения особенностей взаимодействия атомов в функции потенциала инкапсулируются квантовые эффекты. Приблизить результаты моделирования в макроскопическом аспекте оказывается возможным с помощью задания случайных начальных скоростей согласно распределению Максвелла (учет хаотического термодинамического движения). Достоинства метода МД используются для точного определения микроскопических структур материалов, моделирования нанообъектов с целью исследования их свойств и поиска способов синтеза.

В настоящей работе реализация метода МД в составе программного пакета LAMMPS с поддержкой параллельных вычислений [5] использована для моделирования распространения волны горения по образцу в виде пакета чередующихся слоев наноразмерных кристаллических решеток из атомов $\mathrm{Ni}$ и $\mathrm{Al}$ с образованием алюминидов никеля в процессе СВ-синтеза.

Каждый «крупный» слой пакета чередующихся слоев наноразмерных кристаллических решеток из атомов $\mathrm{Ni}$ и $\mathrm{Al}$ (рис. 1) состоит из нескольких атомных слоев (атомных плоскостей), образующих кристаллическую решетку из элементарных кристаллических ячеек типа ГЦК (англ. fсc) с параметрами: для Ni параметр $a=0.3524$ нм, для $\mathrm{Al}$ параметр $a=0.405$ нм [6, 7]. Доля атомов Ni равна $n=0.7975$ (79.75\%), а отношение числа атомов равно $\mathrm{N}_{\mathrm{Ni}} / \mathrm{N}_{\mathrm{Al}}=3.94$. При заданной начальной температуре образца (рис. 1), равной 600 К, производилась «релаксация» всей структуры в течении 0.4 нс с фиксированными термодинамическими параметрами: число атомов $N=717410$, внешнее давление $P=1$ Бар и температура $T=600$ K (NPT-ансамбль). По всем трем измерениям на этом этапе были установлены периодические граничные условия.

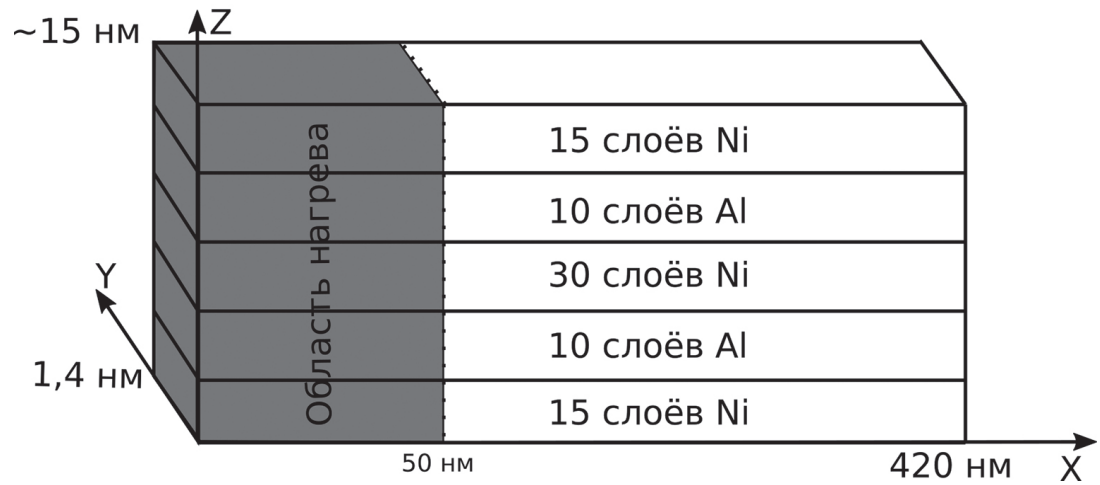

Рис. 1. Схематичное представление исходной слоистой структуры смеси $\mathrm{Ni}-\mathrm{Al}$ 
Для всего образца периодические граничные условия сохранялись и на следующем этапе, на котором в течение 0.1 нс осуществлялся прогрев структуры от 600 до 1200 К в начальной области образца (50x1.4x15 нм) в условиях NVT-ансамбля, где V - объем области нагрева. Для системы атомов в оставшейся области образца (с размерами $370 x 1.4 x 15$ нм) в течение этого же промежутка времени устанавливались условия NVE-ансамбля (E - суммарная энергия атомов). Затем стартовало моделирование распространения волны CB-синтеза с сохранением условий NVE-ансамбля для всего образца. При этом накладывались «свободные» гра- ничные условия на границы расчетной области вдоль оси X, а вдоль осей Y и Z оставались периодические граничные условия.

Две разновидности потенциала межатомного взаимодействия в модели «погруженного атома» (англ. embedded atom model - EAM) использовались в вычислительных экспериментах (ВЭ) по моделированию эволюции системы Ni-Al. B работе [6] в 2002 г. опубликована первая версия потенциала (введем обозначение ЕAM_2002), а вторая версия — в работе [7] 2009 г. (обозначим ЕАM_2009). Результаты ВЭ представлены ниже на рисунках $2,3,4$ и 5.

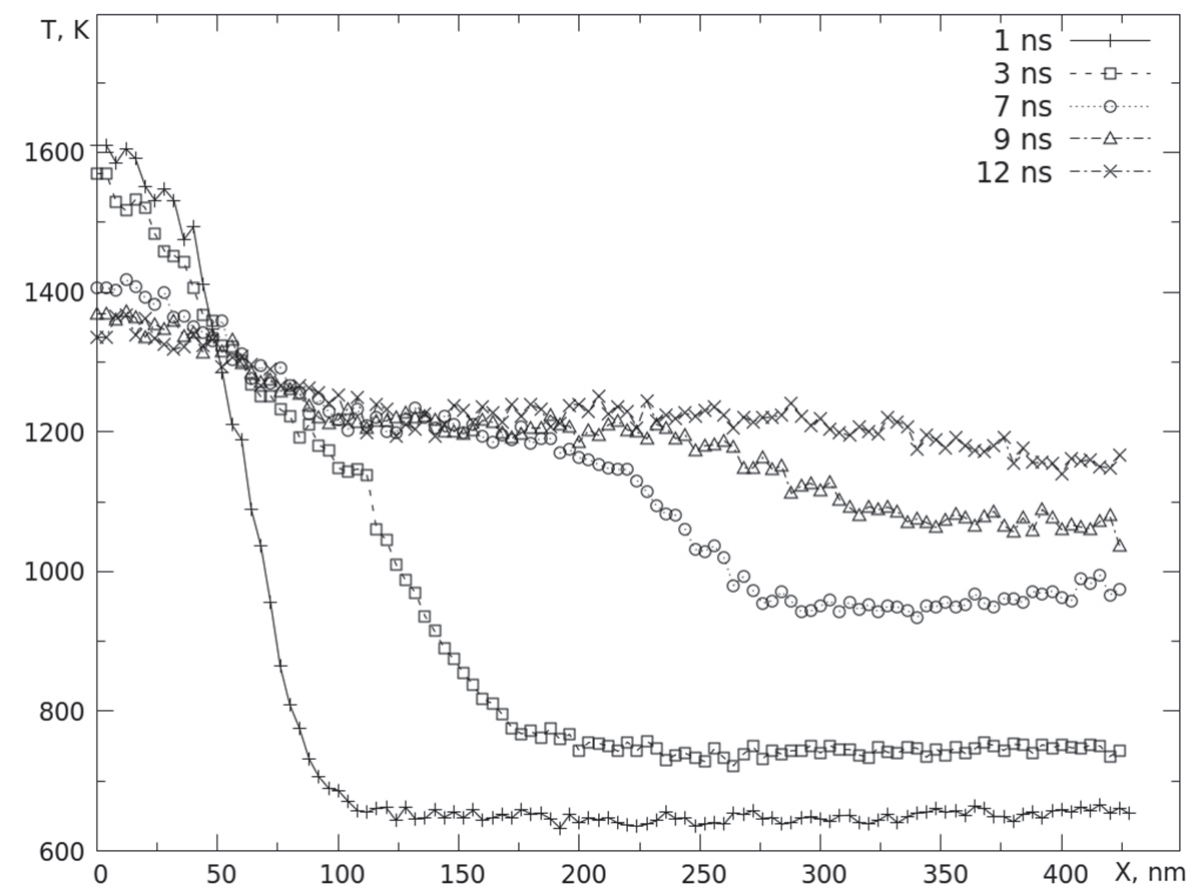

Рис. 2. Семейство температурных профилей как функциональных зависимостей температуры горения СВС от координаты $X$ для последовательных моментов времени (потенциал ЕАМ_2002)

Для потенциала EAM_2002 по границам «плато» каждого температурного профиля (рис. 2) оценивалась скорость движения фронта волны горения, приблизительно равная 35 м/с, т.е. для микро- и наноразмерных образцов слоистых структур (англ. nanofoils) кинетика горения развивается на два порядка быстрее, чем в макрообразцах. Аналогичные условия и схема проведения ВЭ приведены в работе [8], в которой время прогрева начальной зоны составляло 0.08 нс, т.е. на 0.02 нс (или на 25\%) меньше, чем в ВЭ авторов данной статьи. В работе [8] скорость движения фронта волны горения равна 20.13 м/с, что заметно меньше значения 35 м/с (рис. 2). Разницу по ско- рости движения фронта, вероятнее всего, можно объяснить более длительным временем прогрева начальной зоны образца в ВЭ авторов данной статьи. В работе [8] для времени 16 нс граница температурного «плато» горения соответствует $1260 \mathrm{~K}$, которая достаточно близка к соответствующему значению температурного «плато» (приблизительно 1230 К, рис. 2).

Оценка скорости движения фронта волны горения при использовании потенциала EAM_2009 (рис. 3) приблизительно равна 25 м/с, но к концу 12 нс только начинает формироваться температурное плато порядка 1500 К (рис. 3). 


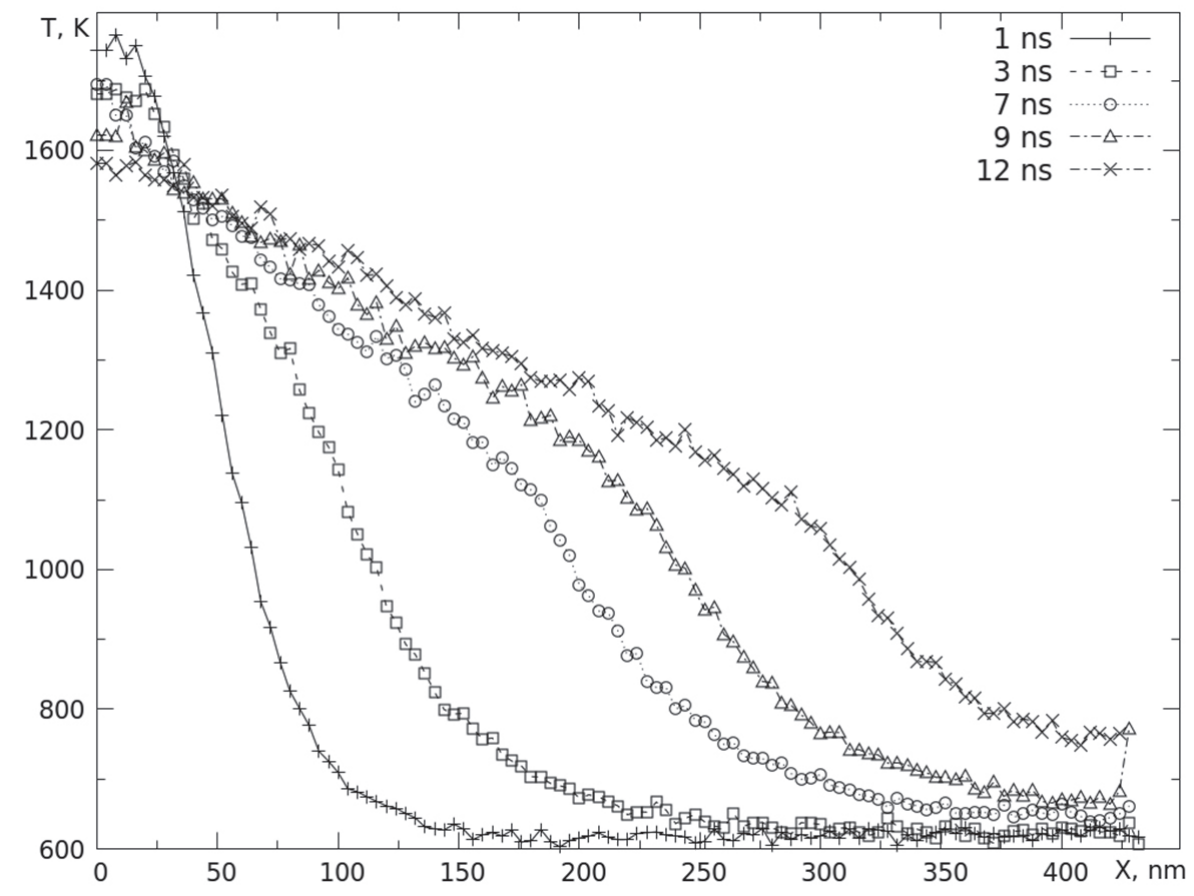

Рис. 3. Семейство температурных профилей как функциональных зависимостей температуры горения СВС от координаты $X$ для последовательных моментов времени (потенциал EAM_2009)

В работе [9] приведены аналогичные условия моделирования ВЭ с тем же временем 0.08 нс для прогрева начальной зоны образца от 600 до $1200 \mathrm{~K}$, как и в [8], но для потенциала EAM_2009. Для начальной температуры релаксации $600 \mathrm{~K}$ (рис. $4 \mathrm{a}$, [9]) и для значения доли атомов $\mathrm{Ni}$, совпадающей со значением $n=0.7975$ (принятым в данной статье авторов), оценка скорости движения волны горения в работе [9] приблизительно равна 20 м/с, которая также меньше 25 м/с (рис. 3), что также можно, вероятнее всего, объяснить большим временем прогрева $(0.1$ нс) начальной зоны образца, установленного в ВЭ авторами данной статьи. В работе [9] для температуры релаксации 600 К (рис. 4b, [9]) данные по температуре горения для $n=0.7975$ отсутствуют.

Используя программный пакет OVITO [10], предназначенный для распознавания и визуализации структур, проведен анализ структуры распределения по образцу атомов $\mathrm{Ni}, \mathrm{Al}$ и фазы $\mathrm{NiAl}$ в последовательные моменты времени (рис. 4 и 5 для двух разновидностей потенциала ЕАМ).

Структуры распределения атомов $\mathrm{Ni}, \mathrm{Al}$ и фазы $\mathrm{NiAl}$ при использовании в ВЭ потенциала EAM_2002 (рис. 4) качественно соответствуют аналогичным структурам, представленным в статье [8]. Эволюция структуры (рис. 4) соответствует режиму растворения и кристаллизации из расплава.

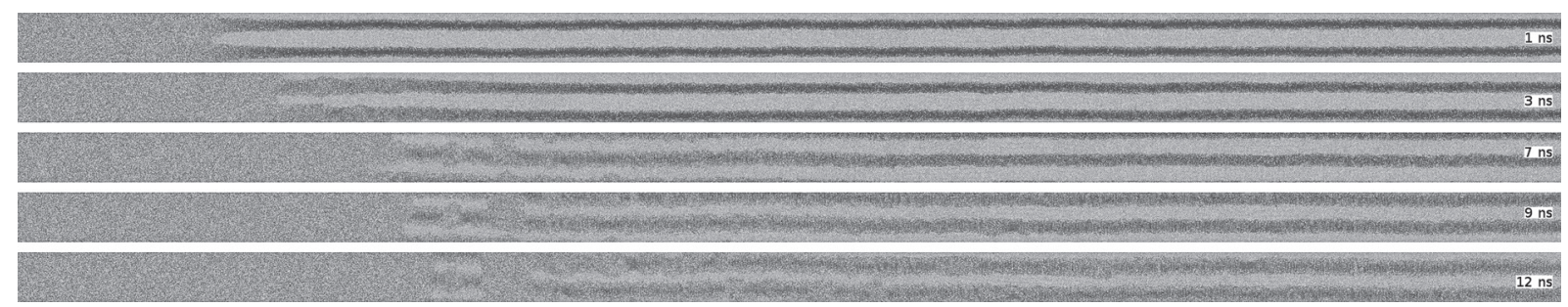

Рис. 4. Набор шлифов (сечений) структуры распределения в слоях атомов Ni (светло-серые точки) и $\mathrm{Al}$ (темно-серые точки) по образцу в последовательные моменты времени: в начале образца однородная зона (слева) соответствует фазе NiAl (потенциал EAM_2002)

Для потенциала EAM_2009 шлифы структур показаны на рисунке 5. 


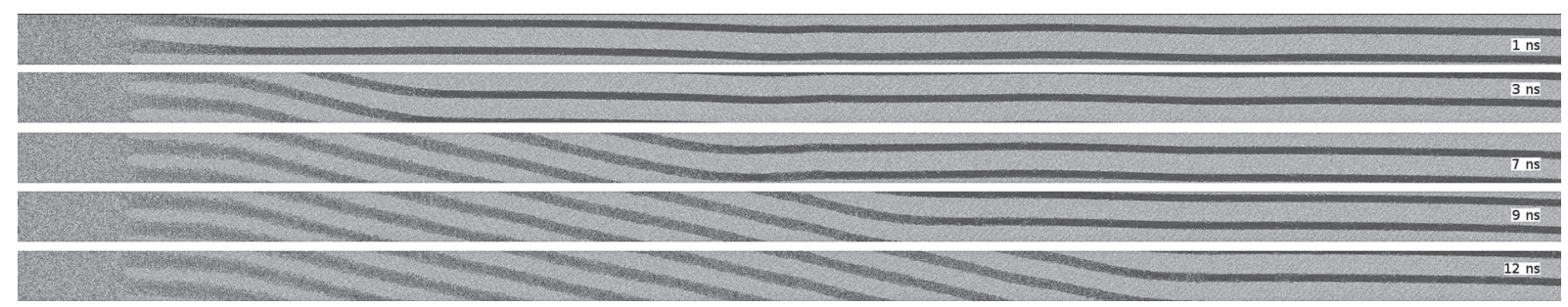

Рис. 5. Набор шлифов (сечений) структуры распределения в слоях атомов $\mathrm{Ni}$ (светло-серые точки) и $\mathrm{Al}$ (темно-серые точки) по образцу в последовательные моменты времени: в начале образца однородная зона (слева) соответствует фазе NiAl (потенциал EAM_2009)

Результаты ВЭ (рис. 5) подтверждают динамику эволюции мозаичной структуры по образцу в последовательные моменты времени, как и в статье [9], которая ссылается на экспериментальное подтверждение мозаичных структур в виде снимков просвечивающей электронной микроскопии (англ. ТЕМ).

Следовательно, модель потенциала EAM_2009 по сравнению с EAM_2002 можно считать более корректной. Мозаичная структура формируется в результате смены режима «растворения и кристаллизации из расплава» на режим «растворения с выпадением осадка в виде твердой фазы»» алюминидов никеля на границах слоев мозаичной структуры (с частичной кристаллизацией алюминидов никеля внутри слоев структуры). Проявления мозаичности обосновываются в статье [9] следующими условиями: $n>0.5$ и температура релаксации должна быть больше 400 К (в нашем случае $n=0.7975$ и температура релаксации равна 600 К).

\section{Заключение}

Исследование и распознавание локальной неустойчивости горения позволяют своевременно осуществлять контроль и управление процессом СВС для обеспечения его стабильности и однородности во всем объеме порошковой смеси, что актуально в практическом применении СВС для разработки 3D-принтеров готовых изделий из металла и металлокерамики в области аддитивных технологий.

\section{Библиографический список}

1. Мержанов А.Г. Твердопламенное горение. - Черноголовка, 2000.

2. Итин В.И., Найбороденко Ю.С. Высокотемпературный синтез интерметаллических соединений. - Томск, 1989.

3. Иордан В.И. Концептуальный подход и комбинированная модель волновой динамики горения с учетом комплекса структурных и многофазных превращений при СВ-синтезе интерметаллических фаз в гетерогенных системах // Известия Алтайского гос. ун-та. Серия: Физика. - 2011. — № 1/2 (69).

4. Jordan V., Kotenev S. Theoretical aspects of mathematical modeling of SHS-process with consideration of the diffusion kinetics and interphase transformations in "mesocells" of heterogeneous powder mixture // J. Communications in Computer and Information Science. - 2014. - V. 487. DOI 10.1007/978-3-319-13671-4_19.

5. Plimpton S. Fast Parallel Algorithms for Short-Range Molecular Dynamics // J. Comp. Phys. - 1995. — Vol. 117.
6. Mishin Y., Mehl M.J. and Papaconstantopoulos D.A. Embedded-atom potential for B2-NiAl // Phys. Rev. B. 2002. - Vol. 65, issue 22. - Article ID 224114.

7. Purja Pun G.P. and Mishin Y. Development of an interatomic potential for the Ni-Al system // Philosofical Magazine. - 2009. - Vol. 89. - Issue 34-36.

8. Politano O., Baras F. Molecular Dynamics Simulations of self-propagating reactions in $\mathrm{Ni}-\mathrm{Al}$ multilayer nanofoils // J. Alloys and Compd. - 2015. - Vol. 652.

9. Turlo V., Politano O. and Baras F. Microstructure evolution and self-propagating reactions in $\mathrm{Ni}-\mathrm{Al}$ nanofoils: an atomic-scale description // J. Alloys and Compd. - 2017. Vol. 708 .

10. Stukowski A. Visualization and analysis of atomistic simulation data with OVITO - the Open Visualization Tool // Modelling and Simulation in Materials Science and Engineering. - 2010. - Vol. 18. - Article ID 015012. 